\title{
TRANSMEDIACIÓN Y TRANSNACIONALISMO: NUEVAS CARACTERÍSTICAS DEL PERIODISMO DE INVESTIGACIÓN EN EL REPORTAJE "TARJETA ROJA"
}

Investigative journalism, transmediation and transnationalism: analysis of the tv reportage "Tarjeta roja"

Jefferson Sanguña

Universidad San Francisco de Quito

jefer.quiroz14@gmail.com

\section{Artículo Científico}

Fecha de recepción del artículo: 10/12/2018

Fecha de aceptación definitiva: 29/12/2018 


\section{Jefferson Sanguña}

\section{Transmediación y transnacionalismo: nuevas características del periodismo de investigación en el reportaje "Tarjeta roja"}

\section{RESUMEN}

En Ecuador, se han realizado libros y tesis sobre el periodismo de investigación. Sin embargo, ninguno de esos trabajos ha visto a ese tipo de periodismo desde los enfoques transnacionales y transmediáticos; tampoco, ninguno de ellos ha abordado la temática deportiva. Por tanto, se realizó una pesquisa para conocer si existieron o no elementos transnacionales y transmediáticos que se hayan incorporado al periodismo deportivo de investigación del país. Todo esto a partir del estudio de caso del reportaje "Tarjeta roja" del programa Visión 360 de Ecuavisa. Se utilizó el análisis de contenido junto con una matriz diseñada a partir de los niveles de interacción que existen en las redes sociales, como parte de la metodología. Para estudiar lo transmediático, se consideraron las plataformas web YouTube y Twitter. Por tanto, luego de la investigación, se determinó la nula existencia de estas técnicas dentro del periodismo de investigación deportivo en Ecuador.

Palabras clave: periodismo deportivo, análisis de contenido, investigación periodística, Ecuador, televisión.

\section{ABSTRACT}

In Ecuador, books and theses on investigative journalism have been made. However, none of these works have seen this type of journalism from transnational and transmedia approaches; neither, none of them has addressed the sports theme. Therefore, a survey was conducted to determine whether or not transnational and transmedia elements existed that have been incorporated into the country's sports research journalism. All this from the case study of the Tarjeta Roja report of the Vision 360 program of Ecuavisa. Content analysis was used together with a matrix designed from the levels of interaction that exist in social networks, as part of the methodology. To study the transmedia, the web platforms were considered: YouTube and Twitter. Therefore, after the investigation was determined the null existence of these techniques in sports research journalism in Ecuador. Keywords: sports journalism, content analysis, journalistic research, Ecuador, television. 
Jefferson Sanguña

\section{Transmediación y transnacionalismo: nuevas características del periodismo de investigación en el reportaje "Tarjeta roja"}

\section{INTRODUCCIÓN}

El periodismo de investigación se convirtió en una especialización del periodismo como tal debido a una serie de características que la diferenciaron del periodismo en general. Al respecto, Juan Carlos Calderón, periodista de investigación, dijo que es "tomar un hecho oculto concreto; mientras más específico, mejor; concentrarse en este, averiguar sus causas, consecuencias, antecedentes y derivaciones, y mostrar cómo afecta esto al lector. He ahí la esencia del periodismo de investigación" $(2005,14)$. Asimismo, esta serie de características aparecieron en la primera década del siglo XX. Una de las primeras investigaciones se dio en 1906 durante el mandato del expresidente Theodor Roosevelt; puesto que las medidas capitalistas que tomó fueron denunciadas por periodistas socialistas; por ello estos fueron señalados como muckrackers (rastreadores de estiércol) por parte del exmandatario.

Años más tarde, se dieron más pesquisas que consolidaron al periodismo de investigación. Una de las más llamativas fue el caso Watergate que se produjo en Estados Unidos en 1972. Esto tras el supuesto asalto que se dio en la sede del Partido Demócrata, lo cual fue desmentido gracias a las investigaciones de los periodistas del Washington Post, Carls Berstein y Robert Woodward, quienes concluyeron que todo fue planeado por el expresidente Richard Nixon (Caminos, 1997).

A su vez, en Latinoamérica, se crearon áreas de investigación en los medios de comunicación debido a las situaciones que pasaron muchos de los países. En Colombia, se creó la Unidad de Investigación en el diario El Tiempo en la década de los setenta. En Argentina, Horacio Vertvinsky fue reconocido por sus notas periodísticas durante la dictadura entre 1989 y 1999. Años más tarde, Perú se unió a esta lista debido a las denuncias que periodistas como Gustavo Gorriti hicieron en contra del expresidente Alberto Fujimori por la serie de actos de corrupción que este cometió.

Para eso, las noticias que salieron de esos países con respecto a temas políticos eran escuchadas en otras naciones del continente. No obstante, no existía todavía un seguimiento a esas noticias. Es decir, técnicas transnacionales que continuaran dichas 
Jefferson Sanguña

\section{Transmediación y transnacionalismo: nuevas características del periodismo de investigación en el reportaje "Tarjeta roja"}

investigaciones desde otras naciones en conexión con los países de origen. Esto se produjo más tarde con la aparición de la tecnología que no solo ayudaría a lo transnacional, sino también a lo transmediático.

De ahí que las nuevas investigaciones realizadas en la actualidad con esos enfoques fueron varias. Entre las más destacados estuvieron: Wikileaks, Panama Papers, FIFAGates y Odebretch. En primer lugar, fueron casos que necesitaron de la ayuda de colegas de otros países para ir publicando las noticias que involucraban a funcionarios en temas de corrupción; y segundo, porque varios medios ampliaban la información en las páginas web con contenidos interactivos que eran parte del término "transmediación". En ese sentido, se puede mencionar el caso del medio Ojo Público, de Perú, donde el director, Óscar Catilla, señaló que haber trabajado en el caso Wikileaks fue una especie de asociación entre dos mundos" (Hidalgo y Torres, 2016). Esto al referirse al trabajo en conjunto que hicieron Julian Assange y los periodistas del diario The Guardian.

En lo que respecta a las investigaciones realizadas en el tema Odebretch, Panama Papers y FIFAGates, en Ecuador, hubo publicaciones transmediáticas que nacieron desde lo transnacional. Entre los medios que adquirieron estas prácticas estuvieron: diario El Universo, los Medios Públicos EP y Plan V. Eso sí, este último fue el que habló sobre el caso de los FIFAGates; sin embargo, hay que señalar que no es un medio deportivo, sino uno que cubre temas políticos en su mayoría. Además, dicho caso de corrupción salió a la luz después del escándalo que envolvió a la Federación Ecuatoriana de Fútbol (FEF) en 2014, año en el que la investigación en los medios deportivos del país fue nula y uno de los pocos reportajes que se realizaron al respecto fue uno de televisión nacional que tenía un enfoque investigativo y no netamente deportivo.

Tras estas nuevas técnicas que se encontraron en dichos casos, fue necesario reconstruir el periodismo de investigación en Ecuador y, en función de esa pesquisa, se analizó la existencia o no de técnicas trasnacionales y transmediáticas. Por tanto, la aparición de medios de comunicación dedicados a la investigación se produjo desde los años cincuenta del siglo XX. No obstante, algunos de ellos se definían como revistas familiares o medios con temáticas políticas y no netamente investigativas. Primero, se comenzó desde la revista Vistazo, misma que nació el 4 de junio de 1957 de la mano de 


\section{Jefferson Sanguña}

\section{Transmediación y transnacionalismo: nuevas características del periodismo de}

investigación en el reportaje "Tarjeta roja"

los empresarios Xavier Alvarado y Rodrigo Bustamante. Dicha revista emitió varios reportajes de profundidad con temáticas políticas como la desaparición de los hermanos Restrepo, la destitución de Abdalá Bucaram, el ataque de Angostura, entre otros. Por ello, la revista ganó varios reconocimientos en su momento (EP02).

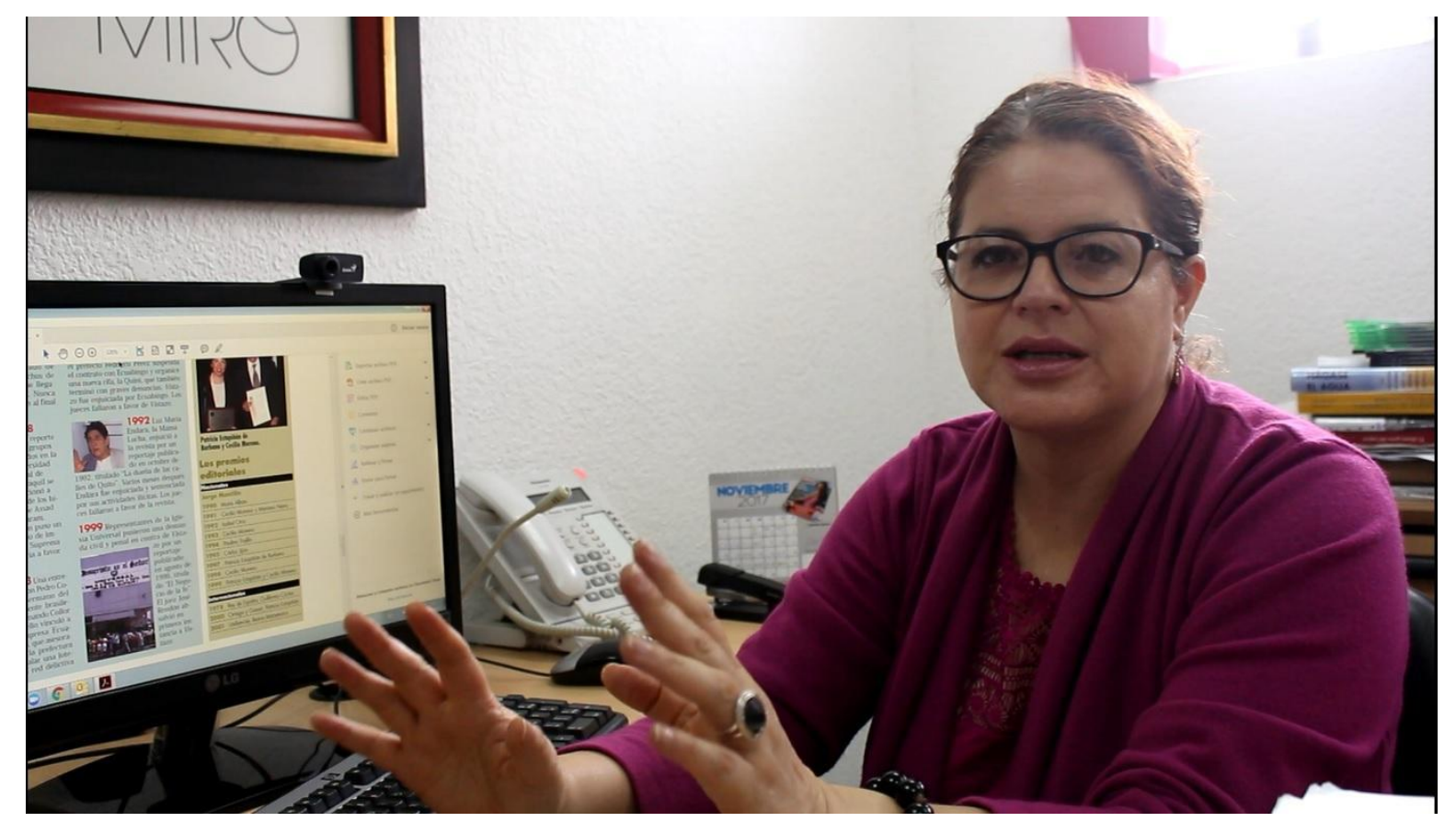

Figura 1: María Belén Arroyo, editora política de la revista Vistazo, explica su trabajo de investigación. Crédito: Jefferson Sanguña.

Luego apareció el programa La Televisión, que nació en enero de 1990 gracias a su fundador Freddy Ehlers, este programa terminó su emisión en diciembre de 2015. Con gran influencia del medio de comunicación Sixty Minutes de los Estados Unidos. Su objetivo fue poner en la pantalla un contenido distinto al que ya se emitía. Para Ehlers, el programa no era netamente de periodismo de investigación; puesto que para él todo periodismo conlleva investigación (EP01).

En ese mismo sentido, pero años más tarde, nació el programa Día a Día que se transmite en Teleamazonas. La creación del programa fue en 1998 y, hasta la fecha, sigue emitiéndose cada fin de semana. Los reportajes que se han emitido han sido de temáticas diversas. Por tanto, Gabriela Osorio, directora del programa, dijo que más que un programa 
Jefferson Sanguña

\section{Transmediación y transnacionalismo: nuevas características del periodismo de investigación en el reportaje "Tarjeta roja"}

de investigación, Día a Día es una revista familiar. Sin embargo, algunos de sus reportajes han sido de profundidad. Uno de ellos fue el que realizaron sobre las escuelas de conducción del país y la corrupción que existía en esos centros (EP03).

Posteriormente, se siguió con libros investigativos realizados por periodistas. Uno de ellos fue El juego del camaleón, los secretos de Angostura, de Arturo Torres, experiodista de diario El Comercio, publicado el 16 de marzo de 2009. El texto fue una recopilación de información sobre cómo las Fuerzas Armadas Revolucionarias de Colombia (FARC) fueron insertándose en el territorio ecuatoriano. La realización de esta pesquisa le llevó aproximadamente siete años durante los que hizo alrededor de 70 entrevistas, la revisión de 2000 documentos y la lectura de 10 libros (Torres, 2009). Por tanto, tras todo el trabajo de investigación que realizó fue incluido en la reconstrucción del periodismo de investigación en el país.

Un año más tarde, otro libro se publicó. Fue El gran hermano que prepararon los periodistas Juan Carlos Calderón y Christian Zurita, publicado el 19 de agosto de 2010. Esta obra trató un tema que se rumoraba en la palestra ecuatoriana: la firma de contratos que el gobierno de Rafael Correa tuvo con las empresas de su hermano Fabricio Correa. Brevemente, en el trabajo de investigación, se mencionaron los juicios que los periodistas tuvieron que enfrentar por parte de las denuncias del exmandatario ecuatoriano. No obstante, este libro fue considerado dentro de la reconstrucción del periodismo de investigación en el último siglo por todo lo que implicó: investigaciones, denuncias y demás (El Universo, 2011).

Años más tarde se dio otro hecho donde la prensa ecuatoriana tuvo incidencia por el trabajo realizado. Este fue el de los Panama Papers. Un escándalo que se dio en abril de 2015 gracias a los miles de documentos que una fuente anónima de la compañía Mossack Fonseca dio al periódico alemán Süddeutsche Zeitung. Aquí había cientos de pruebas que involucraban a funcionarios públicos de cientos de países con la evasión de impuestos gracias a empresas de papel en Panamá. Algunos políticos del país también estuvieron involucrados. Es por ello que gracias a periodistas como Paúl Mena, Mónica Almeida y Xavier Reyes de diario El Universo y Arturo Torres, Andrés Jaramillo y Alberto Araujo de El 
Jefferson Sanguña

Transmediación y transnacionalismo: nuevas características del periodismo de investigación en el reportaje "Tarjeta roja"

Comercio, por nombrar a algunos, se descubrió a varios de los funcionarios y los medios publicaron las noticias (EP04).

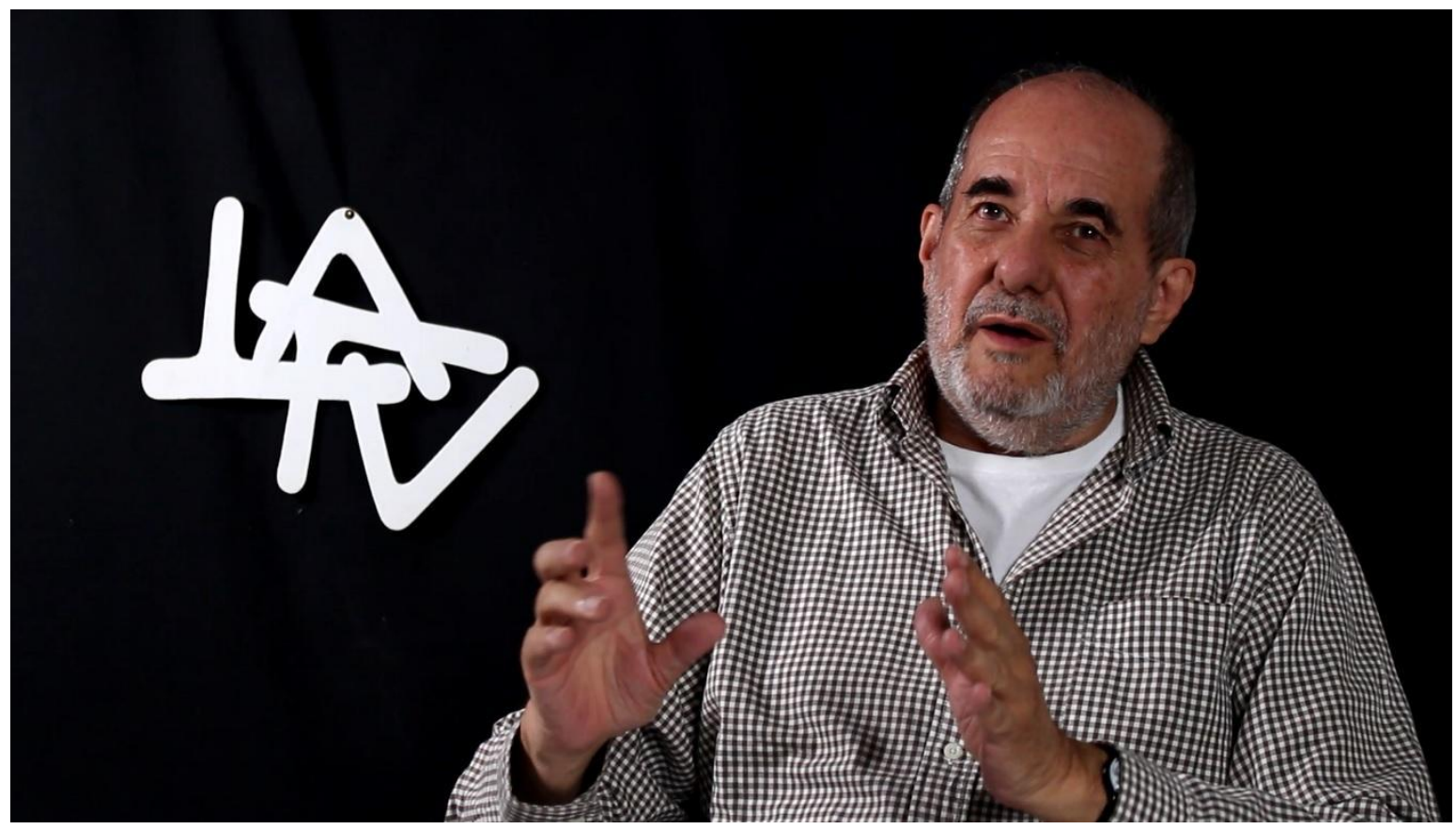

Figura 2: Freddy Ehlers dirigió el programa La Televisión durante 15 años (19902015). Crédito: Jefferson Sanguña.

Meses después; surgió el escándalo de los FIFAGate el 27 de mayo de 2015. Eso a partir de la detención de varios funcionarios de la Fédération Internationale de Football Association (FIFA) por parte del Departamento de Justicia de los Estados Unidos. Básicamente, se les acusó de lavado de dinero y aceptación de coimas para dar los derechos de transmisión de partidos de fútbol a una empresa específica. A su vez, uno de los funcionarios que admitió esas coimas habría sido Luis Chiriboga, expresidente de la FEF. No sería sino hasta 2016, cuando el extitular fue sentenciado a 10 años de prisión tras comprobarse que lavó 6.1 millones de dólares durante su Presidencia en el organismo del fútbol ecuatoriano. Aquí se destacó que los medios deportivos del país no realizaron ninguna investigación sobre el tema, sino que tuvo que ser un programa de investigación que no era netamente deportivo.

Otro de los casos que se colocó en esta reconstrucción fue el de Odebretch. Esta empresa habría sobornado a varios funcionarios de algunos países de Latinoamérica para 


\section{Transmediación y transnacionalismo: nuevas características del periodismo de}

\section{investigación en el reportaje "Tarjeta roja"}

tener los negocios de las construcciones en esos países. Asimismo, Ecuador estuvo involucrado en el tema y diario El Comercio sacó a la luz una lista de 11 delatores que participaron en dichas transacciones. Tras la primera noticia que surgió desde los Estados Unidos el 21 de diciembre de 2016, varias investigaciones se dieron a lo largo de los países involucrados en el tema.

Finalmente está la creación del último medio dedicado al periodismo de investigación en el país. Es decir, el nacimiento de Visión 360 de Ecuavisa, el 30 de marzo de 2014. Actualmente, el programa terminó su cuarta temporada y este sí se califica como un programa exclusivamente de investigación. Además, dichas aclaraciones se han podido evidenciar en los reportajes que han realizado desde su nacimiento como "Tarjeta roja", que se emitió el 31 de agosto de 2014. Este habló sobre la crisis del fútbol ecuatoriano y fue el protagonista para la realización de esta investigación.

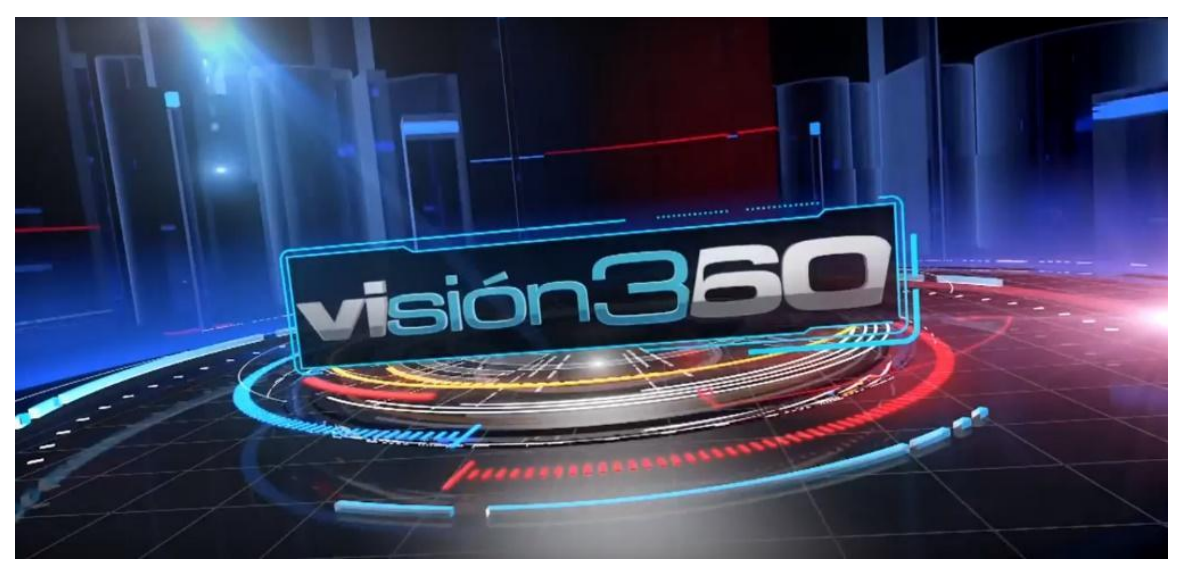

Figura 3: Visión 360 nació como programa semanal de Ecuavisa en 2014. Captura de pantalla.

Todos estos medios realizaron algunos reportajes que empezaron a mostrar tintes trasnmediáticos y transnacionales debido al contenido que mostraron. En ese sentido, fue menester preguntarse qué ocurría en cuanto a la investigación en los medios deportivos. Por tanto, la interrogante que se quiso responder fue qué elementos transmediáticos y transnacionales se incorporaron en el periodismo de investigación deportivo y conocer si dichos elementos crearon nuevas características en el periodismo de investigación. Todo esto se basó en el reportaje "Tarjeta roja" del programa Visión 360 de Ecuavisa. 


\section{Jefferson Sanguña}

\section{Transmediación y transnacionalismo: nuevas características del periodismo de investigación en el reportaje "Tarjeta roja"}

\section{Desarrollo}

El tema de periodismo de investigación en Ecuador ha sido objeto de estudio en algunos casos; por eso, se han realizado varias tesis y libros que hablaron sobre este tema. Al respecto, se pueden mencionar algunas. Primero, estuvo el libro de José María Caminos que se publicó en 1997. Periodismo de investigación: teoría y práctica fue un texto donde se estableció un debate alrededor del término "periodismo de investigación", puesto que unos consideraban que esta era una rama nueva del periodismo y otros creían que era una falacia ya que todo periodismo es de investigación. No obstante, Caminos estableció que sí existe una diferenciación que hace del periodismo de investigación una rama distinta.

Posteriormente, se escribieron libros sobre esa rama de la comunicación. Eso sí, con casos específicos donde se aplicaba dicha arista del periodismo. Uno de esos libros fue el del periodista Arturo Torres que se tituló El juego del camaleón, los secretos de Angostura. Como se mencionó en líneas anteriores, este texto se trató sobre la inserción de las FARC en territorio ecuatoriano. A su vez, se determinó que la muerte de Raúl Reyes fue parte de un proceso de inteligencia y espionaje que se realizó durante varios años. En ese sentido, Torres dio algunas declaraciones donde explicó que, para dar vida a su libro, tuvo que pasar por una serie de investigaciones donde tuvo que encontrar fuentes que hablaran sobre el tema y, además, establecer presupuesto para la realización del mismo (2009).

Por otro lado, se señaló también las pesquisas académicas en cuanto a periodismo de investigación. La tesis de Sharon Domínguez se basó en el estudio de documentales y reportajes con temáticas investigativas. A su vez, Domínguez señala que el caso de los documentales cinematográficos, la creación gráfica dependerá, en gran medida, de la investigación realizada; puesto que, según el trabajo de la autora, ahí es cuando habrá mayor creatividad al momento de producir (2017). Incluso, la autora recalca que todos los reportajes que se muestran en la pantalla tienen una investigación de fondo y agregó que una de las principales características fue generar críticas sociales en la gente y señala que eso provocaron el reportaje Luces, cámara ¿derroche? y el documental La muerte de Jaime Roldós. 
Jefferson Sanguña

\section{Transmediación y transnacionalismo: nuevas características del periodismo de investigación en el reportaje "Tarjeta roja"}

Otro de los estudios consistió sobre la pérdida de investigación dentro del periodismo. En ese sentido, Saudia Levoyer dice que este ha ido desapareciendo por el descrédito que el expresidente Rafael Correa ha emitido sobre los medios de comunicación en general. Además que, por esas razones, a los medios tradicionales "se [les] han cerrado casi todas las puertas para contar y narrar los hechos que ocurren en la esfera de la acción del poder, por causas políticas y económicas" $(2015,4)$. En suma, los espacios para realizar periodismo de investigación se perdieron.

En cuanto al tema de lo transnacional, se pudo encontrar la tesina de Pamela Cruz, misma que habla sobre el retorno del transnacionalismo en el país. Allí explica que ese término es "como un sistema circular de relaciones e intercambios sociales y económicos a través del cual se transmiten conocimientos, información y membresía" $(2013,22)$ No obstante, este estudio tuvo que ver con factores migratorios y no necesariamente periodísticos; a su vez, la inclusión de términos como "información" o "conocimiento" fueron importantes para tomar en cuenta dicho estudio y establecer una relación con la investigación que se realizó.

Por otro lado, fue necesario destacar una tesis que hablaba sobre la investigación y el periodismo deportivo. La autora realizó un análisis de discurso de los diarios El Comercio y La Hora en torno al escándalo de la corrupción en la FIFA (Flores, 2017). Dicha pesquisa se basaba en analizar el discurso y no necesariamente la existencia o no de investigación en el periodismo deportivo; al respecto, fue necesario aclarar que los diarios mencionados ahí son de interés general y no están ligados únicamente a las temáticas deportivas.

Sobre términos usados en la investigación, fue necesario definir cuatro de ellos para este estudio. El primero es periodismo de investigación. Este concepto tuvo varias aristas que mostraron distintas posturas sobre sus significados. Puesto que había ciertos autores que decían que el periodismo de investigación no era más que una falacia, pues argumentaban que todo periodismo tiene investigación y no necesariamente debe ser una especialización. Los autores que más destacaban en torno a esa concepción eran María Pilar Diezhandino, José Javier Muñoz e incluso Gabriel García Márquez. 


\section{Jefferson Sanguña}

\section{Transmediación y transnacionalismo: nuevas características del periodismo de investigación en el reportaje "Tarjeta roja"}

Por otro lado, estuvo la otra cara de la moneda. Es decir que, en efecto, el periodismo de investigación es una especialización que requiere de más tiempo para realizarse. A propósito de ello, la definición con la que se trabajó en este estudio fue la de Juan Carlos Calderón, quien declaró que el periodismo de investigación toma un hecho concreto para averiguar todo lo que lo conlleva; en otras palabras, causas, consecuencias, contexto y derivaciones $(2005,14)$

Tras definir al periodismo de investigación, se analizaron los otros términos posteriormente para establecer la relación que existió entre ellos. El siguiente concepto fue el de transmediación que para esta investigación usó la definición de Campalans et ál. que establecía que la transmediación se refería a aquella forma de comunicación que debía consistir en la interacción, participación e información complementaria que se crearon en las redes sociales o en una plataforma web donde un producto periodístico está plasmado. (Campalans et ál., 2014).

Una vez definido lo transmedia se relacionó con lo transnacional; puesto que esta última se refiere a la comunicación entre varias personas de distintos lugares del mundo. Por lo que en el trabajo de investigación se utilizó la definición de Daniel Mato, quien dice que la transnacionalización es aquella relación de producción que conlleva el apoyo de otras personas que estén ubicadas en diferentes países (2001). Por tanto, este concepto sirvió para esclarecer algunos de los productos periodísticos más sonados, tuvieron que realizarse en conjunto con otros países.

Finalmente, el último concepto fue el de televisión. Puesto que la pesquisa tuvo como centro a un reportaje de ese medio de comunicación en Ecuador. Por eso, la definición que se usó fue la de Rincón y Estrella, quienes dijeron que es un medio de comunicación que tiene la capacidad de alcanzar a varias personas. La televisión, junto con su contenido, ha provocado debates sociales y políticos en función de los contenidos que se han presentado en los mismos (Rincón \& Estrella, 2001) Por tanto, tras las anteriores definiciones, la pesquisa dio algunos resultados sobre el periodismo de investigación deportivo en Ecuador. A su vez, previamente se realizó una reconstrucción de esa rama del periodismo en el país. 


\section{Jefferson Sanguña}

\section{Transmediación y transnacionalismo: nuevas características del periodismo de investigación en el reportaje "Tarjeta roja"}

\section{METODOLOGÍA}

En el transcurso del trabajo investigativo que llevó por título "Periodismo de investigación en la segunda década del siglo XXI: Análisis de las características transmediáticas y transnacionales de las investigaciones deportivas en el programa Visión 360 ", la metodología que se usó fue la descriptiva y el análisis de contenido del reportaje "Tarjeta roja" del programa Visión 360. Entre dos tipos de análisis de contenido: un cuantitativo y un cualitativo, para esta investigación se usó el primero en función de la cantidad de comentarios e interacciones que existieron durante y después de la emisión del reportaje. Por tanto, se explicó el análisis de contenido cuantitativo a partir del autor Berelson. Él dijo que "es una técnica de investigación para la descripción objetiva, sistemática y cuantitativa del contenido manifiesto de la comunicación" (Berelson 1952, en Van Dijk y Rodrigo, 1999, p. 104).

En esa directriz, se añadió lo que Krippendorf dijo sobre dicho análisis. Es decir que esa metodología se fundamentó en el análisis de los datos, el contexto en el que se emitieron, la delimitación de los datos recogidos y el análisis de estos en función de lo que se necesita saber (Krippendorf 1990, en Van Dijk \& Rodrigo-Mendizábal 1999). Además se precisó otra arista que involucró al análisis de contenido. Dicho elemento fue el análisis como estrategia cualitativa. Para esto se estableció la siguiente definición que Strubbs dio al respecto: "Se refiere al intento de estudiar la organización del lenguaje por encima de la oración o la frase y, en consecuencia, de estudiar unidades lingüísticas mayores, como la conversación o el texto escrito" (Strubbs 1987, en Van Dijk \& Rodrigo-Mendizábal 1999, p. 113)

Esto sirvió para interpretar de una manera más concisa la forma en que se exponían los comentarios sobre el reportaje. Asimismo, el contenido de los mismos, los hashtags que usaron en Twitter y YouTube, los "me gusta", la cantidad de comentarios expuestos y el balance que se hizo entre una plataforma y otra. Eso sí, se tuvo en cuenta una cantidad adecuada sobre la base de la recopilación de datos; misma que fue desde la emisión del reportaje hasta el día en que se terminó de usar uno de los hashtags que estuvieron en ambas plataformas. 
Jefferson Sanguña

\section{Transmediación y transnacionalismo: nuevas características del periodismo de investigación en el reportaje "Tarjeta roja"}

No solamente se usó el análisis de contenido como metodología del trabajo investigativo. Otros de los aspectos que se usó fue la entrevista. Esto sirvió para la reconstrucción de la historia del periodismo de investigación en el país. A su vez, fue necesario exponer lo que significa la transcripción de entrevistas para fines investigativos. Al respecto, Rodrigo-Mendizábal señala que se debe hacer un registro concreto sobre el relato. A su vez, dichos relatos pueden ser entrevistas, conversaciones, biografías o historias de vida; mismas que deben ser registradas con la ayuda de medios electrónicos (Van Dijk \& Rodrigo-Mendizábal, 1999).

Luego de haber realizado cuatro entrevistas, se pasó a otro análisis. Puesto que no bastó con transcribir y mostrar lo que los entrevistados dijeron al respecto, sino que fue necesaria una interpretación. A propósito de aquello, Van Dijk y Rodrigo-Mendizábal (1999) explicaron que debe haber un paso connotativo para darle significado a lo que la persona entrevistada dijo. Justamente fue lo que sucedió en la investigación que duró alrededor de cuatro meses. Tras lo declarado por parte de los entrevistados se hizo una connotación al respecto que arrojó datos e interpretaciones sobre la situación del periodismo de investigación deportivo en el país.

\section{ANÁLISIS DE RESULTADOS}

Twitter y YouTube fueron las plataformas web que se analizaron para comprobar la existencia o no de elementos transmediáticos y transnacionales tras la emisión del reportaje "Tarjeta roja" del programa Visión 360 de Ecuavisa. Primero, hubo un preámbulo de estudio en Twitter. En esta red social se precisó que el programa como tal no tiene una cuenta oficial. No obstante, el programa utiliza el hashtag \#Visión360 durante su emisión para que la gente lo utilice y publique sus comentarios respecto al reportaje que se esté proyectando.

La recolección de tuits se basó en dos hashtags que la gente creó al ver el reportaje. Estos fueron \#LárgateChiriboga y \#DemándameChiriboga. A su vez, las publicaciones analizadas se hicieron durante el 31 de agosto de 2014 hasta el 4 de septiembre del mismo año, puesto que la primera fecha indicada fue cuando se proyectó el reportaje y la última mencionada donde se encontraron tuits en relación con "Tarjeta roja". En ese sentido, el análisis se hizo bajo tres niveles: televisivo, transmediático e interactivo. 
Transmediación y transnacionalismo: nuevas características del periodismo de investigación en el reportaje "Tarjeta roja"

Gráfico 1. Tabla de comparación de datos
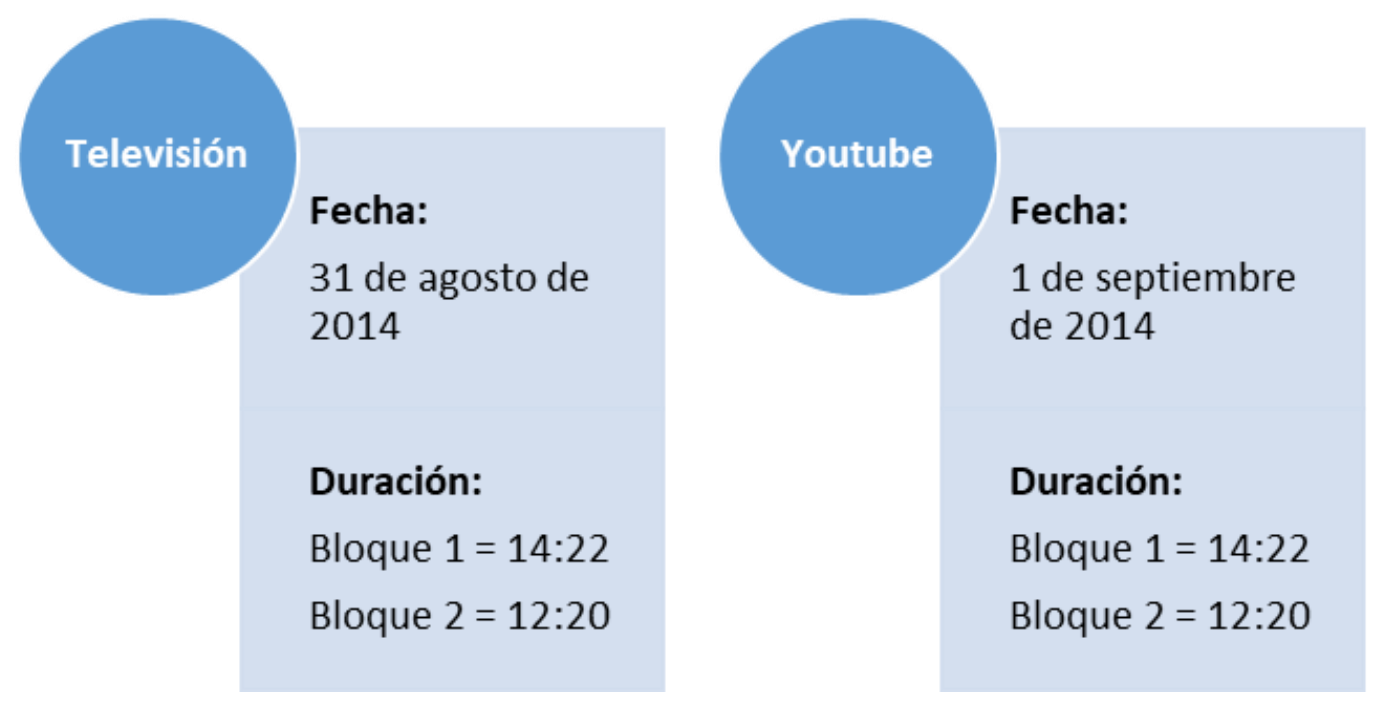

Fuente: Visión 360 Tarjeta roja. Elaboración propia.

En la televisión, se estableció la franja horaria de la transmisión. Es decir, a las 22:30. Asimismo, "Tarjeta roja" tuvo dos partes o bloques, con tiempos de duración distintos. El primer bloque tuvo un tiempo de 14:22, mientras que el segundo, 12:20. En YouTube, el caso fue similar. Al analizar los tiempos de ambas plataformas se evidenció que no cambiaron. Los contenidos subidos a YouTube fueron los mismos que se transmitieron en la televisión. En ese sentido, se pudo evidenciar que YouTube no fue utilizado como herramienta transmediática.

\section{Gráfico 2. Número de visualizaciones en YouTube}

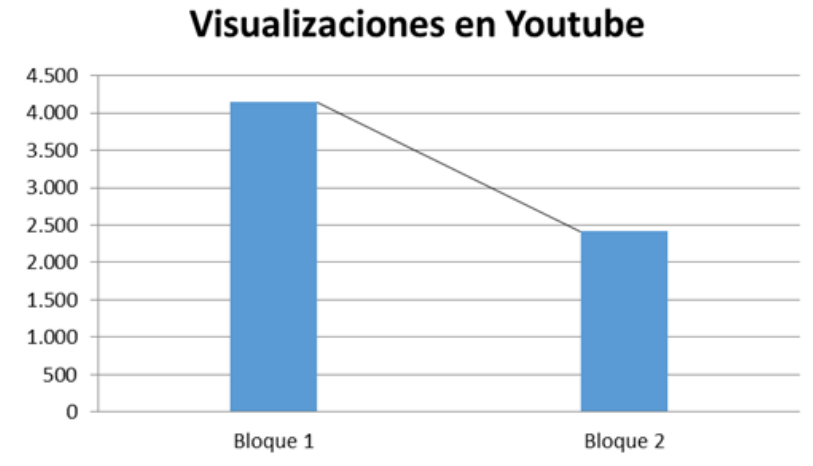

Fuente: Visión 360 Tarjeta roja. Elaboración propia. 


\section{Transmediación y transnacionalismo: nuevas características del periodismo de}

\section{investigación en el reportaje "Tarjeta roja"}

Además, fue menester mostrar el número de visualizaciones, comentarios, "me gusta" y "no me gusta" que existieron. Esto en función del nivel de interacción. En lo que se refiere a las visualizaciones, hubo 4146 en el bloque uno; mientras que, en el segundo, 2416. Además, fue importante mencionar que, en el segundo bloque, fue donde Luis Chiriboga dio aquellas declaraciones donde dijo que seguiría en la Presidencia de la FEF. Esto fue importante para ver cómo los comentarios y tuits se generaron en la otra red social.

Gráfico 3. Número de interacciones entre ambos bloques del reportaje

\section{Interacciones}

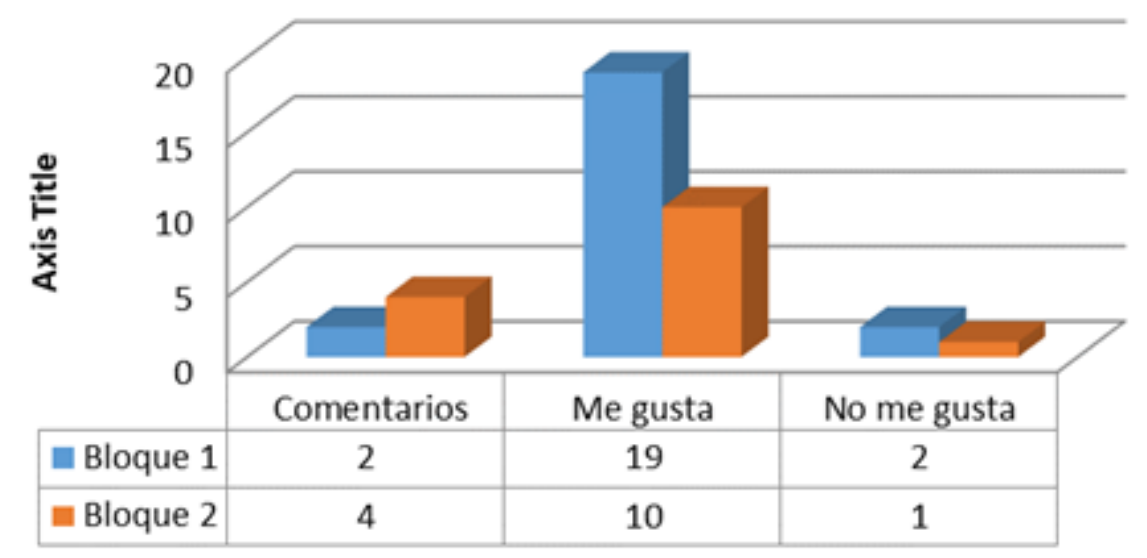

Fuente: Visión 360 Tarjeta roja. Elaboración propia.

Por otro lado, estuvo la comparación entre un bloque y otro en cuanto a interacción. En ese sentido, la primera parte del reportaje tuvo dos comentarios y la segunda, cuatro. En cuanto a "me gusta", el primero tuvo 19 y el otro 10. Finalmente, los "no me gusta" fueron pocos; el primer bloque tuvo dos y el segundo uno. Fue importante destacar que los comentarios que están en YouTube no aportaron mayor información que haya permitido nutrir más al reportaje. Dichos comentarios se basaron en opiniones de las personas; incluso, los usuarios utilizaron los hashtags que también se colocaron en las publicaciones de Twitter.

Posteriormente, se realizó el análisis de Twitter. Aquí fue necesario analizar algunos factores que arrojaron distintos resultados. Primero, se dejó claro que los tuits elegidos fueron del 31 de agosto de 2014 hasta el 4 de septiembre del mismo año. A su vez, esto 


\section{Jefferson Sanguña}

Transmediación y transnacionalismo: nuevas características del periodismo de investigación en el reportaje "Tarjeta roja"

tuvo como resultado una recolección de 100 tuits. De los cuales 89 se publicaron el primer día, cinco en el día dos, dos tuits en los siguientes dos días y el último se publicó solamente uno. Lo que demostró que un $90 \%$ de los tuits se dieron durante la transmisión del programa.

Gráfico 4. Porcentaje de tuits por días

\section{Porcentaje de tuits por día}

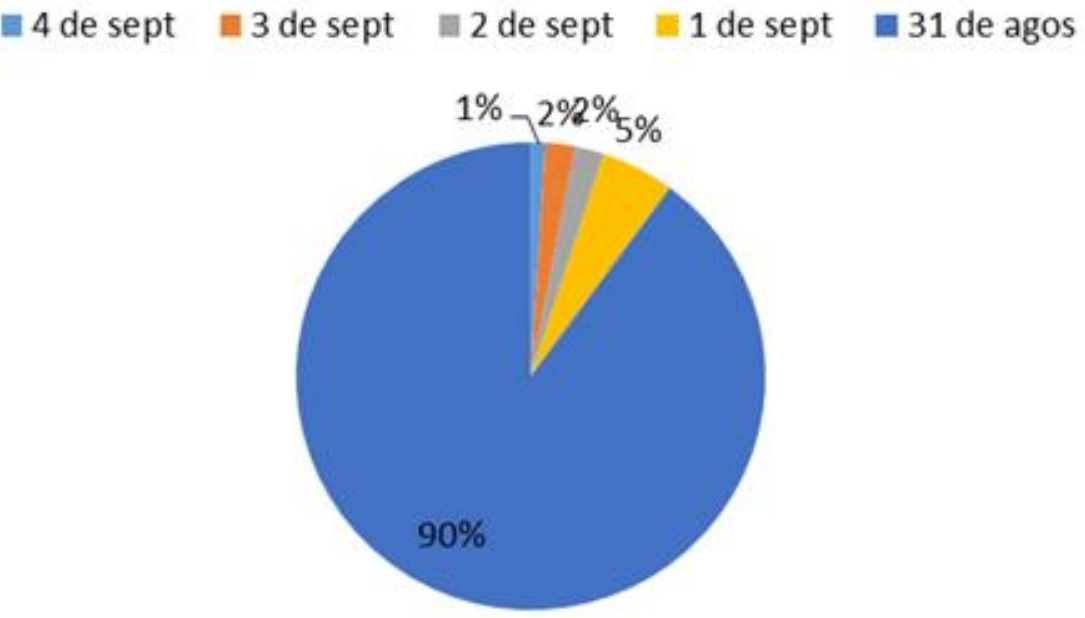

Fuente: Twitter. Elaboración propia.

Por otro lado, estuvieron las interacciones de los tuits que se estudiaron desde tres tipos: la técnica que tiene que ver con los "me gusta", la reactiva con los "retuits" y la mutua con los "comentarios" en los tuits. Asimismo, la interacción que más se usó fue la reactiva; es decir, la de los retuits, que tuvo un total de 148. No obstante, se destacó que el contenido de los mismos no aportaba mayor información. Sino que se basaban en comentarios y opiniones de los usuarios. 
Transmediación y transnacionalismo: nuevas características del periodismo de investigación en el reportaje "Tarjeta roja"

Gráfico 5. Porcentajes de hashtags

\title{
Porcentajes de Hashtags
}

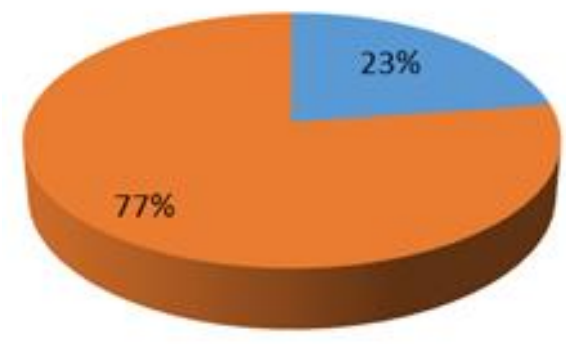

\author{
= \#LárgateChiriboga \\ : \#DemándameChíriboga
}

Fuente: Twitter. Elaboración propia.

Por el lado de los hashtags, se determinó que el más usado fue \#DemándameChiriboga que se escribió en 102 oportunidades que representaron al 77\% y \#LárgateChiriboga fue mencionado en 30 ocasiones, lo que significó un $23 \%$ del total. Finalmente, se mostró cuál fue la cuenta con más interacción y cuál fue la que menos tuvo. Para el primero, la cuenta de Twitter de Ecuavisa fue la que más interacción tuvo con 24 me gusta, 46 retuits y 13 comentarios. Por el contrario, la cuenta que menos interacción tuvo fue el usuario @PASF que no tuvo ningún comentario, retuit o me gusta.

Gráfico 6. Comentarios en YouTube y Twitter

\section{Comentarios}

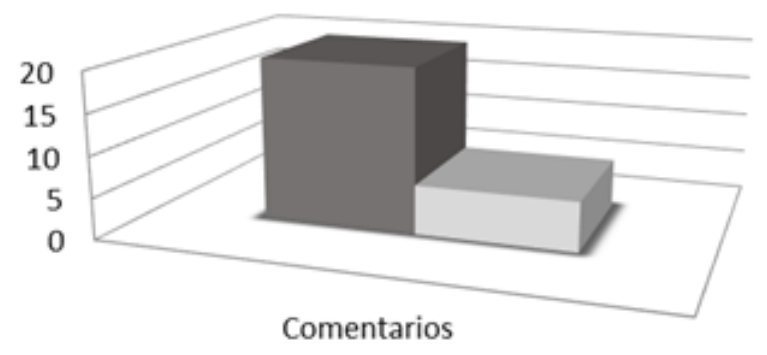

\begin{tabular}{|c|c|}
\cline { 2 - 2 } \multicolumn{1}{c|}{} & Comentarios \\
\hline — Twitter & 20 \\
\hline Y Youtube & 6 \\
\hline
\end{tabular}

Fuente: Visión 360 Tarjeta roja y Twitter. Elaboración propia. 


\section{Transmediación y transnacionalismo: nuevas características del periodismo de} investigación en el reportaje "Tarjeta roja"

Posteriormente, la relación de estudio que se produjo fue entre ambas plataformas web: YouTube y Twitter. Todo esto en función de la interacción. Primero se establecieron las similitudes entre dichas redes. En otras palabras, los comentarios y los me gusta. Lo que respecta a los comentarios, la diferencia fue notoria. Twitter tuvo 20 en total, mientras que YouTube tuvo seis.

Gráfico 7. "Me gusta" en YouTube y Twitter

\section{Likes o Me gusta}

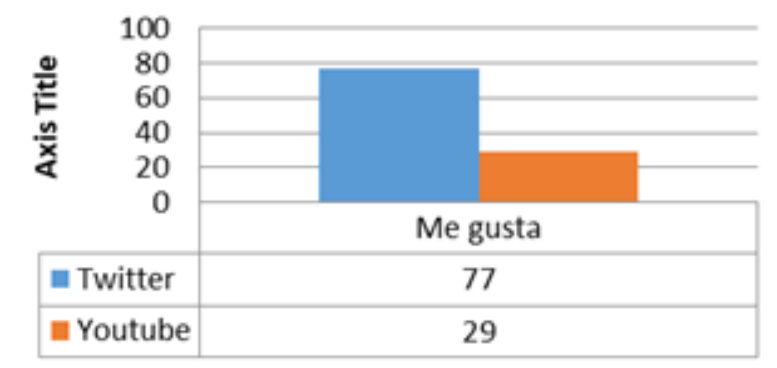

Fuente: Visión 360 Tarjeta roja y Twitter. Elaboración propia.

Finalmente, en los me gusta, Twitter tuvo 77 y YouTube, 29. Como se mencionó antes, ninguna de las interacciones tuvo incidencia en cuanto a la ampliación de la información o contenido adicional del reportaje.

\section{DISCUSIÓN}

Luego del análisis realizado, fue necesario mostrar la relación de los conceptos y los estudios hechos. En lo que respecta a la transmediación, se dijo que esto era el aporte de contenidos ante la nota común y corriente. En otras palabras, adicionar información multimedia con el fin de ampliar la información. En ese sentido, los resultados demostraron que no hubo transmediación, sino transmedialidad. Para entender mejor este último concepto, hay que definirlo. Entiéndase que para Jenkis, la transmedialidad "a diferencia de la transmediación, se caracteriza por el tránsito de un producto mediático a otras pantallas y plataformas para que sea percibida, disfrutada y consumida por audienciasusuarios" (Van Dijk \& Rodrigo-Mendizábal, 1999). 


\section{Jefferson Sanguña}

\section{Transmediación y transnacionalismo: nuevas características del periodismo de}

investigación en el reportaje "Tarjeta roja"

Puesto que en YouTube se colocó el mismo contenido del reportaje que se vio en la televisión, no existió ningún aporte más al video. Por tanto, YouTube fue una herramienta que sirvió para la transmedialidad y no para la transmediación. Además, se pudo denotar la relación entre la interacción y la transmediación en cuanto a Twitter. Se demostró que los comentarios y publicaciones que los usuarios realizaron no tuvieron aporte mayor a la información. En otras palabras, la red social Twitter aportó más a la interacción que a la transmediación.

Por el lado de la televisión, se pudo constatar que, en efecto, es un medio que llega a las personas con contenidos que pueden formar un criterio en ellas. Ahí, se evidenció que esto fue cierto porque durante y después de la emisión del reportaje, la gente expresó su pensamiento por medios de comentarios en ambas plataformas donde el programa tuvo interacción. Es decir, YouTube y Twitter.

Finalmente, en lo que respecta a lo transnacional, se constató que el resultado fue incierto porque esto trata del traspaso de fronteras en cuanto a la producción de una noticia. Sin embargo, el reportaje como tal se realizó desde un mismo sitio sin que se haya necesitado el apoyo de personas extranjeras que trabajaran en el mismo tema. Sino que toda la producción se hizo desde Ecuador por parte de la periodista Carolina Mella y los productores Ramón Murillo y Freddy Pérez.

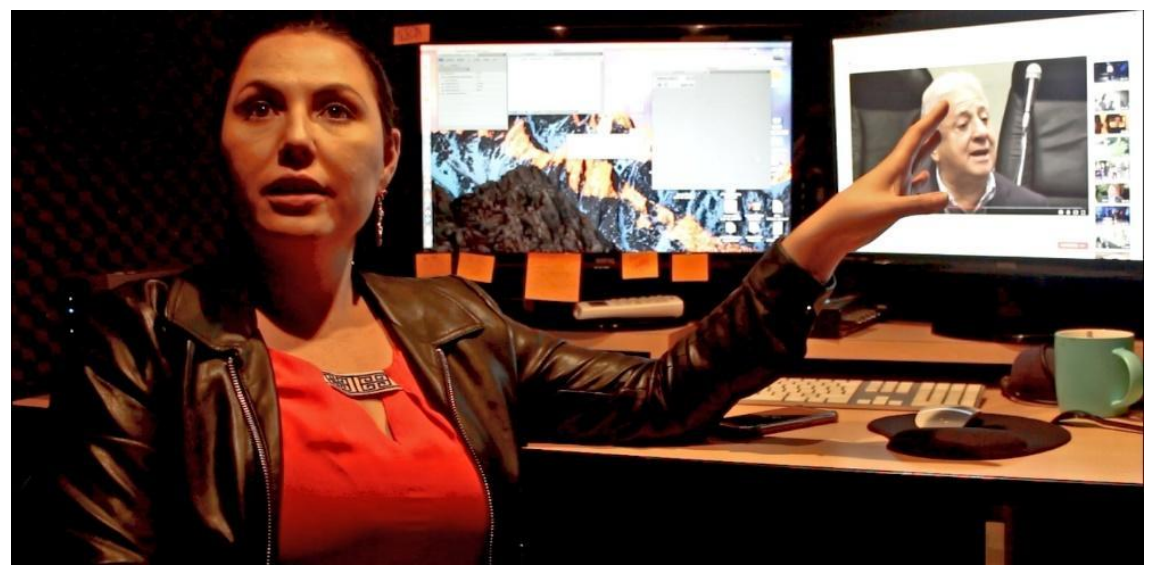

Figura 4: Carolina Mella fue una de las realizadoras del reportaje "Tarjeta roja" del programa Visión 360. Crédito: Jefferson Sanguña. 


\section{Transmediación y transnacionalismo: nuevas características del periodismo de} investigación en el reportaje "Tarjeta roja"

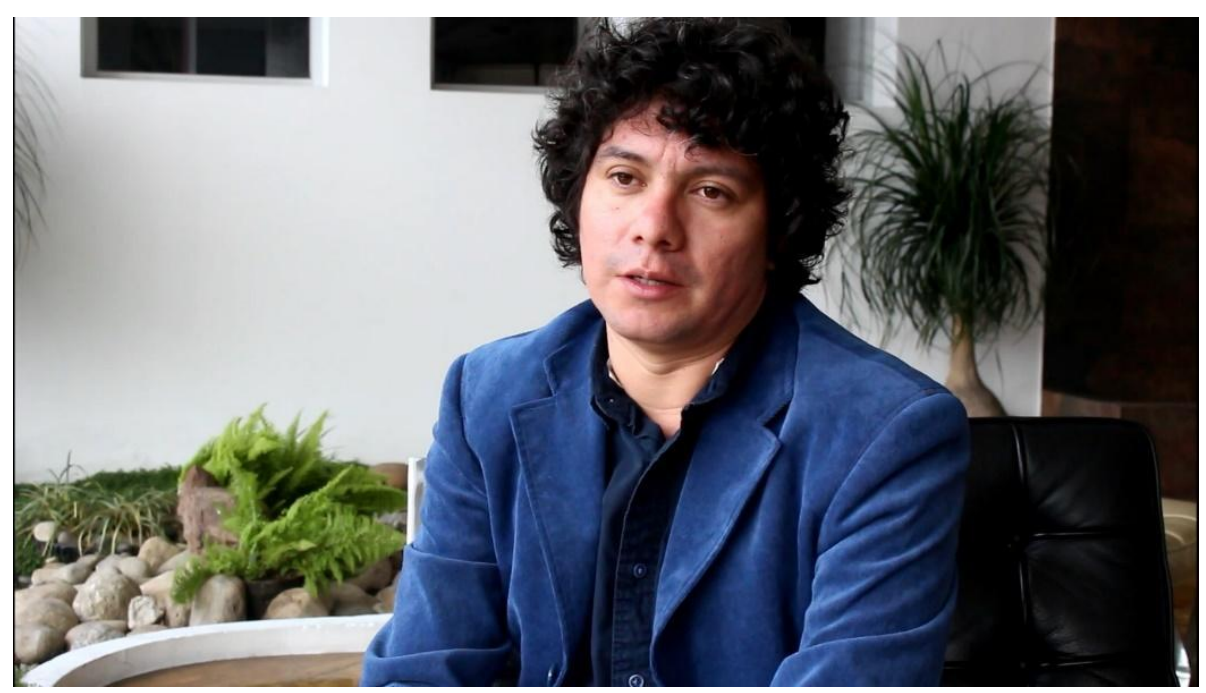

Figura 5: Ramón Murillo estuvo involucrado en la producción del reportaje "Tarjeta roja" del programa Visión 360. Crédito: Jefferson Sanguña.

\section{CONCLUSIONES}

A manera de conclusión, se pudo deducir varios resultados que se obtuvieron luego de la investigación. En primer lugar, se constató que la investigación en el periodismo deportivo no existe. Puesto que durante las denuncias que hubo desde los jugadores por el incumplimiento de sus sueldos, no hubo mayor seguimiento al caso. No obstante, tuvo que ser un programa de investigación como Visión 360 quien hizo una nota al respecto y desembocó en demostrar la crisis que existía en la FEF. En suma, la investigación no fue una característica del periodismo deportivo. A su vez, la historia sobre el periodismo de investigación en el país, se puede ampliar en otras investigaciones futuras. Eso en función de una reconstrucción más minuciosa.

Además, se contestó a la pregunta de investigación porque se pudo constatar que no existieron elementos transmediáticos ni transnacionales en el periodismo de investigación deportivo, pues como se mencionó antes, tampoco hay indicios de indagaciones en dicha rama del periodismo. Lo que sí se pudo constatar es que hubo varios niveles de interacción gracias al reportaje en las plataformas de YouTube y Twitter. Asimismo, la metodología utilizada ayudó a concluir dichas premisas donde, en efecto, no 


\section{Jefferson Sanguña}

\section{Transmediación y transnacionalismo: nuevas características del periodismo de investigación en el reportaje "Tarjeta roja"}

hubo elementos transnacionales ni de transmediación, sino una interacción con tintes de transmedialidad. Es importante mencionar que la matriz usada para la investigación no es la única. Es decir, se puede utilizar otra que ayude a mejorar la recopilación de datos para futuras investigaciones relacionadas a los temas estudiados en el presente texto.

A propósito de lo anterior, no existió ninguna transmediación sino transmedialidad del reportaje "Tarjeta roja" del programa Visión 360. Debido al traspaso de contenido que hubo. Es decir, trasladar el producto audiovisual a la pantalla del internet. Finalmente, es necesario mencionar que, tras las entrevistas realizadas, se pudo conocer que el programa está hecho para la televisión con productos realizados para dicho medio y no pensados en términos de la web.

\section{REFERENCIAS}

Calderón, J. (2005). Periodismo de Investigación. Quito: Editorial Quipus, CIESPAL. Caminos, J. (1997). "Periodismo de investigación: teoría y práctica". En El periodismo de investigación. Madrid: Síntesis.

Campalans, C., Renó, D. y Gosciola V. (2014). Narrativas transmedia entre teorías y prácticas. Barcelona: UOC.

Cruz, P. (2013). Representaciones del migrante retornado: un análisis del discurso de ecuatorianos y ecuatorianas en el contexto de la crisis española. Tesina de posgrado, Facultad Latinoamericana de Ciencias Sociales, Quito, Ecuador.

Domínguez, S.G. (2017). Estado ecuatoriano, periodismo de investigación y cine documental: análisis de los procesos de investigación del documental La Muerte de Jaime Roldós y el reportaje Luces, cámara ¿derroche? Tesis de licenciatura, Universidad San Francisco de Quito USFQ, Quito, Pichincha, Ecuador.

El Universo. (2011). “Autores del libro El Gran Hermano contrademandan a presidente Correa (2011)". El Universo. Obtenido «http://www.eluniverso.com/2011/06/15/1/1355/autores-libro-contrademandancorrea.html.

EP01-Freddy Ehlers, 27 de octubre de 2017. 
Jefferson Sanguña

\section{Transmediación y transnacionalismo: nuevas características del periodismo de} investigación en el reportaje "Tarjeta roja"

EP02-María Belén Arroyo, 27 de octubre de 2017.

EP03-Gabriela Osorio, 20 de octubre de 2017.

EP04-Alberto Araujo, 16 de noviembre de 2017.

Flores, A.Y. (2017). Análisis del discurso emitido por diario El Comercio y La Hora, en relación al escándalo de corrupción de la FIFA, y su influencia sobre la opinión pública generada en torno a la Federación Ecuatoriana de Fútbol. Tesis de licenciatura, Universidad Central del Ecuador, Quito, Pichincha, Ecuador.

Hidalgo, D. y Torres, F. (2016). La navaja suiza del reportero. Herramientas de investigación en la era de los datos masivos. Lima: Ojo Público / Consejo de la Prensa Peruana.

Levoyer, S. (2015). Periodismo de investigación, ¿el espacio perdido en el Ecuador?

Paper Universitario. Obtenido 20 de septiembre de 2017 de 〈http://repositorio.uasb.edu.ec/bitstream/10644/4620/1/CON-PAPLevoyer\%2c\%20S-Periodismo.pdf .

Mato, D. (2002). "Transnacionalización de la industria de la telenovela, referencias territoriales y producción de mercados y representaciones de identidades transnacionales". Ponencia XXIII International Congress of the Latin American Studies Association (LASA), Washington DC, 6-8 septiembre.

Visión 360. (2014, 09 / 01). Tarjeta Roja parte 1| Programa 23 - Bloque 1| Visión 360. Youtube. Obtenido el 28 de noviembre de 2017 de 〈https://www.youtube.com/watch?v=kMJ8hnf5kTc〉.

Visión 360. (2014, 09 / 01). Tarjeta Roja parte 2| Programa 23 - Bloque 1| Visión 360. Youtube. Recuperado el 28 de noviembre de 2017 desde: 〈https://www.youtube.com/watch?v=dL8kCRtsZEM〉.

Rincón, O. y Estrella, M. (2001). Televisión: pantalla e identidad. Quito: Editorial El Conejo

Torres, A. (2009). El juego del camaleón, los secretos de Angostura. Recuperado de 〈http://www.openisbn.com/preview/9978161252/〉.

Van Dijk, T. y Rodrigo-Mendizábal, I. (1999). Análisis del discurso social y político. Quito: Abya-Yala. 
Jefferson Sanguña

Transmediación y transnacionalismo: nuevas características del periodismo de investigación en el reportaje "Tarjeta roja"

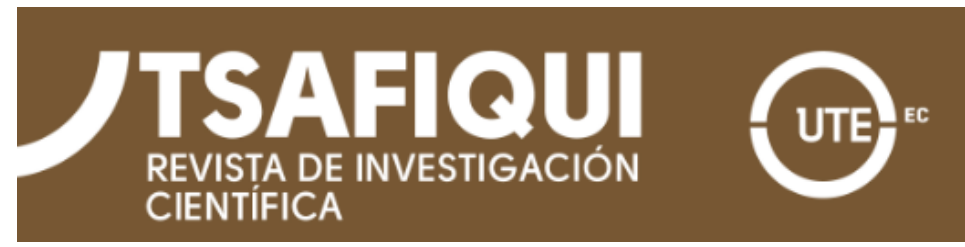

